\title{
Use of Checklist Prevents Errors during Surgeries
}

\author{
1Jatin Kumar, ${ }^{2}$ Akash Sud
}

\section{ABSTRACT}

'Hospitals do most of the right things, on most patients, most of the time.

The checklist helps them to do all the right things, on all patients, all the time.'

According to International Patient Safety Goal-4 (IPSG-4), the hospitals need to ensure the correct patient, correct site, correct procedure and correct surgery. WHO launched Safe Surgery Saves Lives program in 2009 to reduce the number of surgical deaths across the globe. In the complex setting of an operating room, any of the steps may be overlooked during the fast-paced preoperative, intraoperative, or postoperative preparations. So a customized checklist was introduced after sensitization and training of all OT users to reinforce accepted safety practices and foster better communication and team work between clinical disciplines. The aim of the checklist is to reduce the number of errors during surgery and to reduce postoperative complications. The use of the checklist reduced the rate of deaths and complications by more than one-third. The rate of major inpatient complications dropped from 11 to $7 \%$, and the inpatient death rate following major operations fell from 1.5 to $0.8 \%$ after implementation of the checklist. Audit tool printed at the backside of the checklist is analyzed every month, to observe the shortcomings.

Keywords: Surgery, Patient safety, Safe surgery checklist, IPSG 4.

How to cite this article: Kumar J, Sud A. Use of Checklist Prevents Errors during Surgeries. Int J Res Foundation Hosp Healthc Adm 2013;1(1):25-28.

\section{Source of support: Nil}

Conflict of interest: None

\section{INTRODUCTION}

Surgical care and its safe delivery affect the lives of millions of people. About 234 million major operations are performed worldwide every year and $>1$ million of these individuals die from complications. Surgical care has been an essential component of health care worldwide. There are 3 central problems in surgical safety - unrecognized as a public health issue, lack of data on surgery and outcomes and Failure to

\footnotetext{
${ }^{1} \mathrm{Head},{ }^{2}$ Medical Superintendent

${ }^{1}$ Department of Quality, Fortis Hospital, Noida, Uttar Pradesh India

${ }^{2}$ Fortis Hospital, Noida, Uttar Pradesh, India

Corresponding Author: Jatin Kumar, Head, Department of Quality, Fortis Hospital, B-22, Sector 62, Noida-201301, Uttar Pradesh, India, e-mail: js1104@gmail.com
}

use existing safety know-how. As the incidences of traumatic injuries, cancers and cardiovascular disease continue to rise, the impact of surgical intervention on public health systems will grow. There are between 1500 and 2500 wrong site surgery incidents every year in the US. ${ }^{6}$ In a survey of 1050 hand surgeons, $21 \%$ reported having performed wrong-site surgery at least once in their career. ${ }^{6}$

To assist operating teams in reducing the number of these events, WHO Patient Safety—in consultation with surgeons, anesthetists, nurses, patient safety experts and patients around the world-has identified ten essential objectives for safe surgery. ${ }^{1}$ They are:

(1) Operate on the correct patient at the correct site. (2) Use methods known to prevent harm from administration of anesthetics, while protecting the patient from pain. (3) Recognize and effectively prepare for life-threatening loss of airway or respiratory function. (4) Recognize and effectively prepare for risk of high blood loss. (5) Avoid inducing an allergic or adverse drug reaction for which the patient is known to be at significant risk. (6) Consistently use methods known to minimize the risk for surgical site infection. (7) Prevent inadvertent retention of instruments or sponges in surgical wounds. (8) Secure and accurately identify all surgical specimens. (9) Effectively communicate and exchange critical information for the safe conduct of the operation. (10) Hospitals and public health systems will establish routine surveillance of surgical capacity, volume and results.

The checklist identifies three phases of an operation, each corresponding to a specific period in the normal flow of work: before the induction of anesthesia ('sign in'), before the incision of the skin ('time out') and before the patient leaves the operating room ('sign out'). In each phase, the circulating nurse must confirm that the surgery team has completed the list. ${ }^{1,4}$

\section{MATERIALS AND METHODS}

Execution of a customized version of WHO Safe Surgery checklist in all OTs was planned. Training programs for all the surgical staff was planned. A Steering Committee comprising of surgeons, anesthetists, technician, nurses, administration and quality department was formed which later transformed into implementation team. Role of implementation team was divided into 3 broad categories modification of current checklist, awareness and training, retrospective 
and prospective data collection. The surgical safety culture survey forms were circulated to all the OT personnel. Filled forms were collected, analyzed and discussed. It was decided that safe surgery checklist to be considered as a continuous quality improvement tool outside the medical record. Next an online survey was conducted for the staff that missed the first round of survey. Training of nurses, technicians, surgeons and anesthesiologists were conducted. Small group sessions with individual departments were planned. Also 'Incident Report Committee' was reconstituted. A list of possible postsurgical complications were prepared which included 30-day mortality, postoperative blood transfusion of 4 or more units within 72 hours, pulmonary embolism, stroke, infection of surgical site, ${ }^{7}$ cardiac arrest requiring CPR, myocardial infarction, major disruption of wound, unplanned return to OR, coma of $>24$ hours, unplanned intubation, sepsis/septic shock/SIRS, acute renal failure, ventilator use for 48 hours or more, VAP, vascular graft failure.

In order to implement the checklist during surgery, a single person must be made responsible for performing the safety checks on the list. It was decided that this designated checklist coordinator will often be a circulating nurse.

The checklist divides the operation into three phases, each corresponding to a specific time period in the normal flow of a procedure - the period before induction of anesthesia, the period after induction and before surgical incision, and the period during or immediately after wound closure but before removing the patient from the operating room. In each phase, the checklist coordinator must be permitted to confirm that the team has completed its tasks before it proceeds onward. As operating teams become familiar with the steps of the checklist, they integrate the checks into their familiar work patterns and verbalize their completion of each step without the explicit intervention of the checklist coordinator. Each team seek to incorporate use of the checklist into its work with maximum efficiency and minimum disruption while aiming to accomplish the steps effectively.

All steps to be checked verbally with the appropriate team member to ensure that the key actions have been performed. Therefore, before induction of anesthesia, the person coordinating the checklist verbally review with the anesthetist and patient (when possible) that patient identity has been confirmed, that the procedure and site are correct and that consent for surgery has been given. The coordinator will visualize and verbally confirm that the operative site has been marked (if appropriate) and will review with the anesthetist the patient's risk of blood loss, airway difficulty and allergic reaction and whether an anesthesia machine and medication safety check has been completed. Ideally the surgeon be present during this phase as the surgeon may have a better idea of anticipated blood loss, allergies, or other complicating patient factors. However, the surgeon's presence is not essential for completing this part of the checklist.

Before skin incision, each team member introduces him or herself by name and role. If already partway through the operative day together, the team can simply confirm that everyone in the room is known to each other. The team confirms out loud that they are performing the correct operation on the correct patient and the correct site and then verbally review with one another, in turn, the critical elements of their plans for the operation, using the checklist for guidance.

They also confirm that prophylactic antibiotics have been administered within the previous 60 minutes and that essential imaging is displayed, as appropriate. Before leaving the operating room, the team reviews the operation that was performed, completion of sponge and instrument counts and the labeling of any surgical specimens obtained. It also reviews any equipment malfunctions or issues that need to be addressed. Finally, the team discusses key plans and concerns regarding postoperative management and recovery before moving the patient from the operating room.

Having a single person leading the checklist process is essential for its success. In the complex setting of an operating room, any of the steps may be overlooked during the fast-paced preoperative, intraoperative, or postoperative preparations. Designating a single person to confirm completion of each step of the checklist can ensure that safety steps are not omitted in the rush to move forward with the next phase of the operation. Until team members are familiar with the steps involved, the checklist coordinator will likely have to guide the team through this checklist process.

A possible disadvantage of having a single person lead the checklist is that an antagonistic relationship might be established with other operating team members. The checklist coordinator can and should prevent the team from progressing to the next phase of the operation until each step is satisfactorily addressed, but in doing so may alienate or irritate other team members. Therefore, hospitals must carefully consider which staff member is most suitable for this role and empower the designated staff.

\section{RESULTS AND ANALYSIS}

The goal of checklist is not role recitation or to frustrate workflow. The checklist is intended to give teams a simple, efficient set of priority checks for improving effective teamwork and communication and to encourage active consideration of the safety of patients in every operation performed. In a survey of 100 (30 doctors, 40 nurses, 
30 technicians), $78.6 \%$ mentioned the checklist was easy to use. $79.2 \%$ says the checklist has improved operating room safety while $21 \%$ are not sure and $18.3 \%$ say the checklist takes a long time to complete. $84.3 \%$ says communication has improved through use of the checklist. Finally $78.2 \%$ say, If I were to be operated, I would surely want the checklist to be used.

Wrong site draped in one surgery, radial pack gauze missing, graft size not prepared, blood not arranged for a surgery, allergy not mentioned in preoperative checklist and evaluation form, antibiotic prophylaxis not given before time out, before sign in, the need to secure central IV access not evaluated, are among the few errors prevented by the use of the checklist. Doing this has improved the chances of detection of possible errors and has helped the unit in implementing timely interventions to prevent serious outcomes. Hence the checklist solves two purposes: ensuring consistency in patient safety and introducing (or maintaining) a culture that values achieving it.

\section{Impact on Financials}

Implementation of checklist leads to: (1) less SSI due to timely administered of antibiotics, 1 hour prior to surgery, ${ }^{8.9}$

(2) decreased ALOS, (3) decreased rate of return to OT,

(4) elimination of error mitigates chances of litigation and compensation.

\section{Impact on Patients}

(1) Financial savings due to decrease ALOS on account of decrease complications, decrease SSI, decrease duration of surgery. (2) Better outcome on account of (a) better planning, safer anesthesia, (b) timely administration of correct medication and elimination of errors and (3) patient safety.

\section{Impact on Employees}

(1) Team work and better communication, (2) smoother and quicker processes, (3) decreased turnaround time, (4) skill enhancement, (5) improved staff morale and (6) secure working environment.

To build a culture of safety in our health care organizations, health care leaders must ensure that, in their organizations, 'incidents', such as surgical mishaps must be routinely reported without the fear of unjust retribution on the part of the unfortunate healthcare worker. When people are not afraid to report adverse incidents because of the existence of a 'just culture' where, when things go wrong, no one is immediately assigned blame (blame culture), only then will we be able to collect accurate and honest data about incidents, analyse them and learn important lessons from them and, in the process, improve our systems and processes so that the care that we provide will be safer. ${ }^{3}$

\section{DISCUSSION}

The checklist should be modified to account for differences among facilities with respect to their processes, the culture of their operating rooms and the degree of familiarity each team member has with each other. However, removing safety steps because they cannot be accomplished in the existing environment or circumstances is strongly discouraged. The safety steps should inspire effective change that will bring an operating team to comply with each and every element of the checklist.

Modification of the checklist should be undertaken with a critical eye. Surgeons, anesthetists, and nurses should be involved in the modification process and the resulting checklist tried in simulated and real-life situations in order to ensure its functionality. ${ }^{2}$ Actively seeking inputs from nurses, anesthetists, surgeons and others is important not only in helping to make appropriate modification but also in creating the feeling of 'ownership' that is central to adoption and permanent practice change.

Monitoring and evaluation of outcomes is an essential component of surgical care. Many facilities and departments already engage in this process; additional data collection is neither recommended nor encouraged if such a system is already in place and proves useful to the clinicians and staff as a means of improving the quality of care. However, in hospitals where results of surgical care are not routinely tracked and postoperative complications are not recorded, or where surveillance mechanisms are not sufficient to identify poor practices, patient safety checklists become law in Nevada. Now government is picking up the baton.

\section{CONCLUSION}

The aim of this checklist is to reinforce accepted safety practices and foster better communication and teamwork between clinical disciplines. ${ }^{5}$ The checklist is intended as a tool for use by clinicians interested in improving the safety of their operations and reducing unnecessary surgical deaths and complications. Advantages of using a checklist are customizable to local setting and needs, Deployable in an incremental fashion, Supported by scientific evidence and expert consensus, Evaluated in diverse settings around the world, Ensures adherence to established safety practices, minimal resources required to implement a far-reaching safety intervention.

The goal of the surgical safety checklist is to help insure that teams consistently follow critical safety steps and thereby minimize the most common and avoidable risk that 
endanger lives and safety of surgical patients. It evaluates the current state of safety practices and standards at our facility and identifies area for improvement.

Use of the surgical safety checklist has demonstrably improved compliance with basic standards of surgical care in diverse hospitals around the world. While the relationship between adherence to standards and decreases in complication rates is likely multifactorial, improving the safety and reliability of surgical care can save lives and promote confidence in the health system.

Leave aside the other advantages, if 2 minutes taken to read aloud one sheet of paper could prevent wrong surgeries (both on wrong patients and on wrong site), it would be more than worth its weight in gold. The moral, legal and financial cost of wrong surgery is unquantifiable.

\section{REFERENCES}

1. World Alliance for Patient Safety. WHO guidelines for safe surgery. Geneva: World Health Organization 2009.
2. Safe surgeries save life initiative. Implementation Guidelines. Quality in Medical Care Section, Medical Development Division, Ministry of Health Malaysia, Nov 2009 guidelines.

3. Gawande AA, Thomas EJ, et al. The incidence and nature of surgical adverse events in Colorado and Utah in 1992. Surgery 1999;126:66-75.

4. Haynes AB, Weiser TG, et al. A surgical safety checklist to reduce morbidity and mortality in global population. $\mathrm{N}$ Eng $\mathrm{J}$ Med 2009;360;491-499.

5. Weiser TG, Regenbogen SE, Thompson KD, et al. An estimation of the global volume of surgery: a modeling strategy based on available data. Lancet 2008;372;139-144.

6. Seiden, Archives of Surgery, 2006.

7. Joint Commission, Sentinel Event Statistics, 2006.

8. Bratzler. The surgical infection prevention and surgical care improvement projects: national initiatives to improve outcomes for patients having surgery. Am J Surg 2006.CID 2006:43, 328.

9. Classen DC, Evans RS, Pestotnik SL, Horn SD, Menlove RL, Burke JP. The timing of prophylactic administration of antibiotics and the risk of surgical-wound infection. New England J Med 1992a;326(5):281-286. 